\title{
A BeTter WAy For Finding THE OPTIMAL NuMBER OF NODES IN A DISTRIBUTED DATABASE MANAGEMENT SYSTEM
}

\author{
${ }^{1}$ Rashed Mustafa, ${ }^{1} \mathrm{Md}$. Javed Hossain and ${ }^{2}$ Thomas Chowdhury \\ ${ }^{1}$ Department of Computer Science and Telecommunication Engineering \\ Noakhali Science and Technology University, Bangladesh \\ ${ }^{2}$ Department of Computer Science and Engineering \\ Chittagong University of Engineering and Technology (CUET), Bangladesh \\ E-mail: rashed_mustafa78@yahoo.com,javed_abc@yahoo.com and thms_chy@yahoo.com
}

\begin{abstract}
Distributed Database Management System (DDBMS) is one of the prime concerns in distributed computing. The driving force of development of DDBMS is the demand of the applications that need to query very large databases (order of terabytes). Traditional ClientServer database systems are too slower to handle such applications. This paper presents a better way to find the optimal number of nodes in a distributed database management systems.
\end{abstract}

Keywords: DDBMS, Data Fragmentation, Linear Search, RMI.

\section{Introduction}

Now a day, commercial data is increasing rapidly. Due to technological innovation, the size/price of storage devices improves briskly. For business and scientific purposes, sometimes, data size touches the thousandterabyte limit. To process and to keep such a big databases, the demand of supercomputing has risen over twelve years. But the price of the parallel system is very high and the price of Personal Computer (PC) has fallen and its performance has been increased tremendously. So, recently, developing a Distributed system has become popular.

This paper is organized as follows: section 2 briefly discussed about distributed database management system, in section 3 proposed strategy has been analyzed, section 4 discusses its analytical result and finally section 5 conclude the paper which concentrates future work also.

\section{Distributed Database Management System (DDBMS)}

In a DDBMS, data are scattered in several nodes for security, faster processing or any other business purposes. For retrieving information it needs querying of data. Most applications require searching operation than inserting or updating a data to a database. To design a Distributed Database system for such applications, this research proposes to consider optimal number of nodes for ensuring the search faster.

The main disadvantages of a DDBMS are network overhead, process startup time and database connectivity time [1]. To simplify our following discussion we express these disadvantages as Distributed System Overhead (DSO) [2]. For small amount of data, a distributed database (DDB), which consists of hundreds of node, suffers huge network congestion. As a result, the performance will degrade. On the other hand for large amount of data (terabytes), the performance of a distributed database system, which or system that consists of few nodes, may also degrade due to heavy load in each node [4-6].

The above discussion promote that, number of nodes is a prime concern to design a DDBMS. This research identifies an optimistic number of nodes of such a system. There are two approaches to store tuples in the DDBMS [7].

\subsection{Replication}

Identical replicas of the whole database are stored in each node. This storage system increases the robustness of database [5]. But the main disadvantage of this storage system is that is takes a huge amount of disk space to store the data. Also, as the system needs to ensure that all replicas of a relation $r$ must be consistent so whenever $\mathrm{r}$ is updated, the update must be implemented to all nodes of the system. Thus update increases overhead 
in replication storage system. But it enhances the read-only operations [8].

\subsection{Fragmentation}

Data can be fragmented vertically and horizontally. Horizontal fragmentation splits the relation by assigning each tuple of $r$ to one or more fragments. Whereas vertical fragmentation splits the relation vertically, i.e. one more relation will be created from a relation $[9,10]$.

\section{Theory and Analysis}

In worst case, the complexity of linear search algorithm, $f(n)=N, N$ is number of data in an array, where's the particular element is being searched.

If the comparison time of the element is $t_{c}$, then the total comparison time required for worst case in linear search is $N \times t_{c}$.

If $N$ is very big and the data is kept in several nodes of a network, the comparison time for each node will be,

$T_{d}=(N / P) \times t_{c}, P$ is the number of nodes.

One demerit of the distributed system is that we can't omit DSO. Suppose, the time taken to initialize the network for each node is $t_{n}$, the time for linear search in a distributed database is

$T_{d}=\left((N / P) \times t_{c}\right)+P t_{n}$

If $\mathrm{P}=1$ i.e. the search is sequential then according to Equation (1), the comparison time will be

$T=\left(N \times t_{c}\right)+t_{n}$

There're two part of Equation (1), $(N / P) \times$ $t_{c}$, this part represents the time taken for a comparison in each node. It will be decreased if we introduce more nodes without increasing the amount of data. On the other side, $P t_{n}$ is $D S O$, suffered by the application. It will be increased if we introduce more nodes without increasing the amount of data.

Now, we take a look in Equation (1), if we introduce more nodes in the system, more DSO will be introduced. But it will decrease comparison time i.e. the searching will be optimal in the DDB only when

$(N / P) \times t_{c}=P t_{n}$

From Equation (3), we can write that the optimal number of nodes for worst case will be

$P=\sqrt{\frac{N t_{c}}{t_{n}}}$
And for average case it will be

$$
P=\sqrt{\frac{N t_{c}}{2 t_{n}}}
$$

Here, $P$ is the number of nodes, $N$ is the amount of data, $t_{c}$ is single compare time, $t_{n}$ is network overhead. So, if we know $N, t_{c}, t_{n}$ we can easily find out $P$, the number of node in the distributed system for optimum linear searching.

\section{System Architecture}

For practical purpose, we establish a distributed system using 16 nodes and one workstation, which act as application server. All nodes with identical configuration consist of 128 RAM, 16 GB HDD, P-II Processor with $450 \mathrm{MHz}$. A 100-based D-Link DES1024 fast Ethernet switch with 16K memory is used for network. Operating system (OS) of all nodes and workstation is Windows 2000. MySQL and java RMI are used for distributed database and distributed processing respectively [12].

\section{Proposed Strategy}

Here, at first, we have taken results using $1,2,4,8,10,12,16$ nodes according and, it considers 100,000,000 rows in a table where the data has been searched. The data is kept in the nodes by round robin fashion [11].

We measured the comparison time of each node is $0.81 * 10^{-3} \mathrm{~ms} /$ compare. The startup costs (Initialize Database + Network overhead) $=982 \mathrm{~ms}$. So, from (5) theoretically, $P=9.09$

\section{Result Analysis}

Analytical result shown in Table 1. From table 1 , it is decided that the value of the $P$ lies between 8 and 10. So, we have also taken data for 9 nodes. Table 2 shows the result. From the above results, we see that the number of nodes should be 9 for the best performance and this is equal to our theoretical result.

The following two concepts are useful in comparing sequential and distributed process [2].

The speedup, $S p=T_{s} / T_{d}$

Which is the ratio of the time taken for the optimal sequential algorithm and the time taken required by the proposed distributed algorithm to solve the same problem. 
Table 1 Represents the time taken (Seconds) in each node

\begin{tabular}{|c|c|c|}
\hline Number of Nodes & Number of tuples per Nodes & Time Taken (Second) \\
\hline 1 & $100,000,000$ & 81.75 \\
\hline 2 & $50,000,000$ & 42.03 \\
\hline 4 & $25,000,000$ & 27.54 \\
\hline 8 & $12,500,000$ & 18.56 \\
\hline 10 & $10,000,000$ & 19.65 \\
\hline 12 & $8,333,333$ & 22.44 \\
\hline 16 & $6,250,000$ & 25.29 \\
\hline
\end{tabular}

Table 2 Time taken for 9 nodes

\begin{tabular}{|c|c|c|}
\hline Number of nodes & Number of tuples per Nodes & Time Taken (Second) \\
\hline 9 & $11,111,111$ & 17.83 \\
\hline
\end{tabular}

Which measures the fraction of time that a case of 9 nodes, time is minimum, which typical node (processor) is usefully supports our experimental and also employed.

The following table 3 depicts speedup versus efficiency for corresponding nodes.

Table 3: Calculation of Speedup and Efficiency.

\begin{tabular}{|c|c|c|}
\hline No of nodes & Speedup & Efficiency \\
\hline 1 & 1 & 1 \\
\hline 2 & 1.95 & 0.975 \\
\hline 4 & 2.968 & 0.742 \\
\hline 8 & 4.405 & 0.551 \\
\hline 9 & 4.585 & 0.509 \\
\hline 10 & 4.1603 & 0.416 \\
\hline 12 & 3.643 & 0.304 \\
\hline 16 & 3.233 & 0.2021 \\
\hline
\end{tabular}

The following figures described graphically on the basis of the results of Table 1 and 2. theoretical analysis.

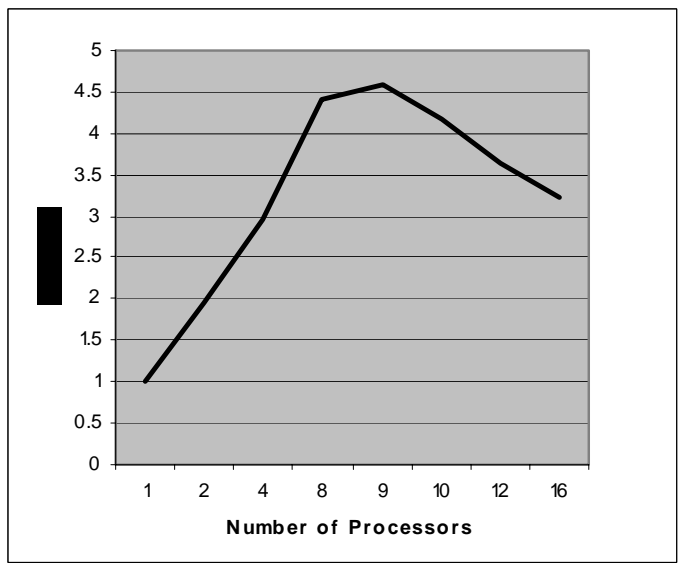

Fig. 2 Number of processors versus speedup ratio

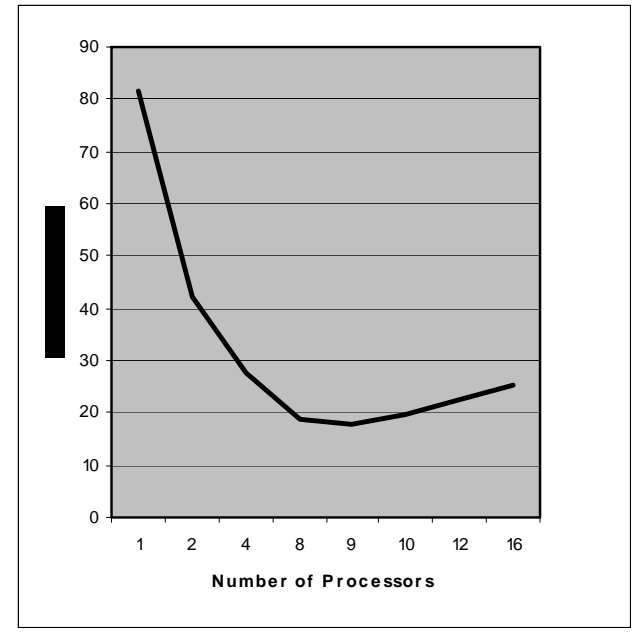

Fig. 1 Number of Processors Vs Time Taken (Second)

Figure 1 depicts that time decreases due to large number of nodes used, but the result is not always proportional to number of nodes. It is clearly shown on the graph that in the
Figure 2 described number of nodes versus speedup ratio. It is shown that speedup ratio goes upward when small number and unoptimized (excluding 8 and 9) number of nodes used.

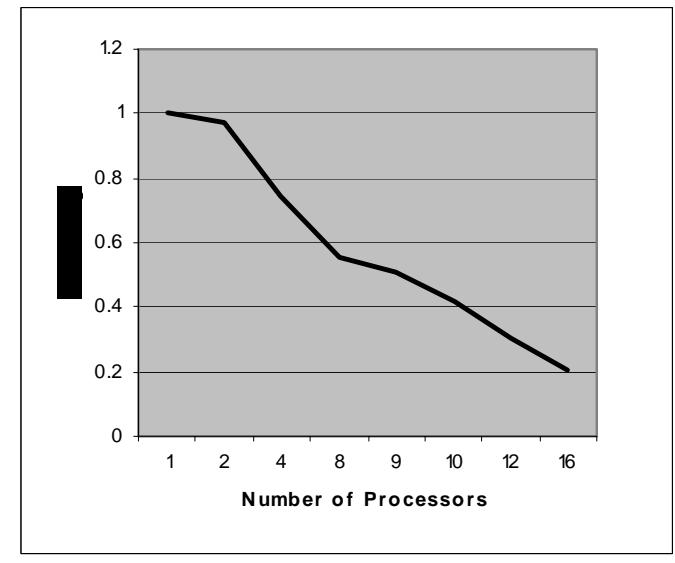

Fig. 3 Number of processors vs. efficiency 
Figure 3 elucidate efficiency in accordance with number of nodes. Here also clearly shown that efficiency is inversely proportional to number of nodes.

\section{Conclusion}

Distributed Database is a growing technology. A huge research work has been done on it and is continuing. In this paper, theoretically and experimentally, we have shown a way to find out the optimal number of nodes for linear search in a distributed database. A related work has been done [3], which does not show the particular number of nodes for optimal searching. In this paper it is clearly identified a number of optimistic nodes for a particular searching.

\section{References}

[1] M. Adelgurefi and K.F. Wong. "Parallel Database Techniques, IEEE-CS press june 1998 (ISBN 0-8186-8398-8)"

[2] D. DeWitt, and J. Gray "Parallel Database Systems: The Future of High Performance Database Systems", CACM, Volume 35, No. 6, June 1992.

[3] D. DeWitt, and J. Gray "Parallel Database Systems: The Future of High Performance Database Systems”, CACM, Volume 35, No. 6, June 1992.
[4] Nowshaba Durrani, Mohammed Anwer, A comparison of parallel database search algorithm on a 16 node cluster, ICCIT 2004, Brac University, Dhaka.

[5] 5. Annaratone, M., Pommerell, C., and Ruhl, R. (1989) Interprocessor communication speed and performance in distributed-memory parallel processors. IN $16^{\text {th }}$ Annul Symposium on Computer Architectures, pp. 315-324, June 1989.

[6] M.B. Ibiza-Espiga and M.H. Williams. "Data placement strategy for a parallel database system" in proceedings of DEXA'92 Conference, pages 469-474, Valencia, Spain, September 1992.

[7] D. Gibson, J.M Kleinberg, and P. Raghavan. Clustering categorical data: An approach based on dynamical systems, In proc. 1998 Int. conf. Very Large Databases (VLDB’98), pages 311-323, New York, Aug, 1998

[8] Java as a Basis for Parallel data Mining, In proc. $7^{\text {th }}$ Intl conf. On High Performance Computing and Networking HPCN Europe, April 12-14, 1999, Amsterdam, The Netherlands, LNCS 1593, Springer verlag, pp.884-884

[9] Silberschatz, Korth and Sudarshan, Database System Concepts, $4^{\text {th }}$ ed., McGraw-Hill, pp.710712, 2002.

[10] Dimitri P. Bartsekas and John N. Tsitsiklis, Parallel and Distributed Computation: Numerical Methods, Prentice-Hall, pp. 27- 28,1989.

[11] Buya R. "High performance Cluster Computing", Vol-1,2, Prentice Hall PTR.

[12] Sun Microsystems: Remote Method Invocation specification,http://java.sun.com/products/jdk/rmi 